rev.relac.int.estrateg.segur.10(1):203-224,2015

\title{
INVERSIÓN EN EL POSCONFLICTO: FORTALECIMIENTO INSTITUCIONAL Y RECONSTRUCCIÓN DEL CAPITAL SOCIAL•
}

\author{
Jairo J. Niño Pérez ${ }^{* *}$ \\ Camilo Andrés Devia Garzón ${ }^{* * *}$
}

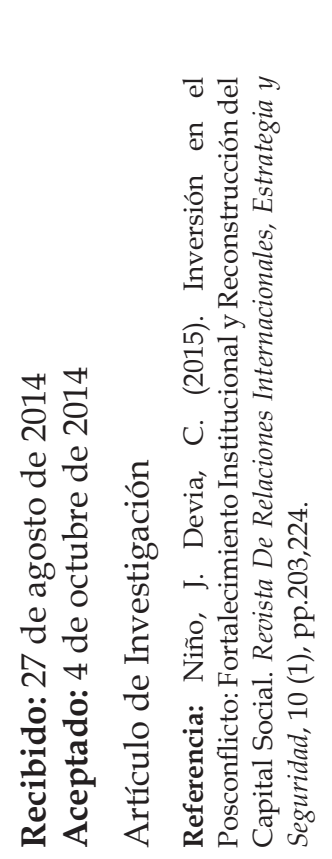

\section{RESUMEN}

Con el fin de la Guerra Fría, los conflictos de carácter intraestatal escalaron en número, duración e intensidad; demandando una reorganización de los objetivos de los agentes internacionales interesados en la reconstrucción subsiguiente. El diagnóstico apropiado de las dinámicas

* Este trabajo es producto del proyecto INV-EES-1559 "Comportamiento de las estructuras criminales de soporte en escenarios de posconflicto en Centro América y África" adscrito a la Facultad de Relaciones Internacionales, Estrategia y Seguridad, financiado por la Universidad Militar Nueva Granada, Bogotá-Colombia vigencia 2014.

** Profesional en Relaciones Internacionales y Estudios Políticos de la Universidad Militar Nueva Granada. Co Investigador del grupo Estudios Internacionales y Políticos de la Facultad de Relaciones Internacionales, Estrategia y Seguridad de la UMNG. Profesional de Marketing y Planeación en la Dirección de Estrategia de Telefónica Colombia. jaironpz@gmail.com.

*** Profesional en Relaciones Internacionales y Estudios Políticos, Maestría en Asuntos Internacionales, Universidad Externado de Colombia. Docente investigador, Facultad de Relaciones Internacionales, Estrategia y Seguridad, Universidad Militar Nueva Granada. camilo.devia@ unimilitar.edu.co. 
de inversión y la respuesta del sector privado a los esfuerzos que en esta materia hacen instituciones locales e internacionales, es de importancia capital para optimizar los procesos de reconstrucción de la vida política y económica en escenarios de posconflicto. El presente documento explora esta dinámica con foco en las variables que generan un ambiente propicio para el fortalecimiento institucional y la construcción de capital social, áreas más vulnerables en los conflictos civiles y que desde 1989 han cobrado especial relevancia en los esfuerzos para la construcción de una paz duradera.

Palabras clave: Capital Social, Clima de Inversión, Fortalecimiento Institucional, Inversión, Reconstrucción Posconflicto, Sector Privado.

\title{
INVESTMENT IN POST-CONFLICT: INSTITUTIONAL STRENGTHENING AND RECONSTRUCTION OF SOCIAL CAPITAL
}

\begin{abstract}
With the end of Cold War, intra state civil conflicts grew in number, length and intensity; demanding a new priority scheme in the objectives of international agents interested in postconflict reconstruction. An appropriate diagnose of investment dynamics, and the response of private sector to the efforts made in this regard by both local and international institutions, is a core issue in order to optimize the reconstruction process of the economic and political life in post-conflict scenarios. This document explores those dynamics focusing on the core variables that define the enabling environment to strengthen institutions and building social capital, grouping them into 4 categories known to be essential in the effort to avoid cyclical violence in civil conflicts. These variables: Socioeconomic Stability; Infrastructure; Public Engagement and Institutional Consolidation are central to a proposed model of organization and optimization for resource allocation in peace building efforts.
\end{abstract}

Keywords: Social Capital, Investment Climate, Institutional Strengthening, Investment, Post Conflict Reconstruction, Private Sector.

\section{INVESTIMENTO NO POSCONFLICTO: FORTALECIMENTO INSTITUCIONAL E RECONSTRUÇÃO DO CAPITAL SOCIAL}

\section{RESUMO}

Com o fim da Guerra Fria, os conflitos intra-estatais de caráter escalado em número, duração e intensidade; exigindo uma reorganização dos objetivos dos atores internacionais envolvidos na reconstrução posterior. O correto diagnóstico da dinâmica de investimento e de resposta 
do sector privado para os esforços nesta área tornam as instituições locais e internacionais, é de suma importância para otimizar o processo de reconstrução da vida política e econômica em cenários pós-conflito. Este artigo explora essa dinâmica com foco nas variáveis que criam um ambiente propício para o fortalecimento institucional ea construção de capital social, agrupando-os em quatro tipos considerados essenciais no esforço para prevenir a reativação do ciclo de violência em conflitos civis. Tais variáveis: estabilidade econômica; Infra-estrutura; Envolvimento Público e Fortalecimento Institucional; são a espinha dorsal de um modelo de base para planos de ação estruturação e otimização de recursos para os objectivos a curto, médio e longo prazo no caminho da construção de uma paz duradoura.

Palavras-chave: Capital Social, Clima de Investimento, Fortalecimento Institucional, Investimento, Reconstrução Pós-Conflito, Setor Privado.

\section{INTRODUCCIÓN}

En un escenario de posconflicto, la generación de empleo, la provisión de servicios públicos y la reconstrucción del capital social son tres áreas esenciales en donde las políticas de inversión deben favorecer la creación de incentivos económicos a sabiendas de que resultan fundamentales para abordar algunas de las secuelas que tradicionalmente llevan a una recaída en el conflicto.

El análisis propuesto consta de tres secciones en las que se describen los factores determinantes en el proceso de transformación de una economía de guerra a una economía del desarrollo; con el objetivo de plantear un modelo de organización de las tareas clave para la construcción de la paz en función de una apropiada valoración de las prioridades, y; los objetivos de corto, mediano y largo plazo.

En la primera sección, se abordan las definiciones clave del estudio, describiendo los efectos más importantes del cambio en la preeminencia de guerras entre Estados a conflictos de carácter intra-estatal después de 1990. Aspecto que es fundamental para la investigación en virtud a que exhibe las diferencias entre los efectos sociales causados por los conflictos tradicionales entre Estados y aquellos causados en los escenarios de conflicto civil de que nos ocupamos.

La segunda sección, profundiza en la actividad del sector privado y su rol central en la cimentación de la paz, considerando la forma en que los negocios entre individuos y organizaciones son un elemento que trasciende como vínculo social y cuya caída en un espiral de informalidad, beneficia la conformación y permanencia de estructuras criminales que limitan el desarrollo económico y pueden llevar en el mediano plazo a la reactivación de la violencia. 
Por último, se presenta un modelo a partir de la evolución esperada de las dimensiones críticas en el tránsito del conflicto al desarrollo, que servirá como orientación para encargados de política pública y otros agentes interesados en esta problemática para estructurar planes de acción; definir prioridades y optimizar recursos de cara a los objetivos de corto, mediano y largo plazo.

\section{DEFINICIONES CLAVE}

El presente documento se desprende de la investigación acerca del comportamiento de las estructuras criminales de soporte en un escenario de posconflicto. Dichas estructuras, son aquellas conformadas por agentes desligados de la motivación declarada por los actores del conflicto, pero que participan animados por el lucro proveniente de actividades de provisión de elementos de guerra, drogas, logística de transporte, entre otros necesarios para continuar las hostilidades.

Siendo África parte del marco geográfico de la investigación macro, es también un escenario recurrente en el presente análisis por lo que resulta conveniente plantear aquí un argumento central al estudio: Los procesos de violencia cíclica que son frecuentes en África subsahariana no son considerados un producto de las características de fragmentación cultural o étnica distintivos de esa región del mundo.

Por el contrario, los conflictos civiles intraestatales y la inestabilidad son un fenómeno presente en diferentes regiones del mundo y las tensiones étnicas son un resultado, antes que una causa del conflicto.

En efecto, los ciclos de violencia-paz-violencia son el resultado de la degradación del capital social que se da cuando las instituciones, especialmente aquellas con las que se identifica una autoridad pública, colapsan y los grupos humanos retroceden hacia formas primarias de identificación, reforzando estructuras básicas de solidaridad y favoreciendo un escenario de polarización social en el que las tensiones étnicas emergen (Mikailof, Kostner, \& Devictor, 2002).

Este punto es particularmente importante en el proceso de eliminar sesgos de trasfondo histórico o cultural que pueden limitar la capacidad del observador para identificar la importancia de la afectación de las instituciones y los vínculos sociales en escenarios violentos (Acemoglu \& Robinson, 2012).

Para la opinión pública, las cifras relativas al número de víctimas mortales, personas desplazadas o el costo de la infraestructura destruida, son los mecanismos predilectos para dimensionar el alcance de las hostilidades, determinando en gran medida la intensidad con que los actores involucrados demandan acciones del Sistema Internacional y sus agentes. 
Si bien es cierto que este tipo de indicadores dan cuenta del alcance de los mecanismos y los medios de los actores en conflicto y sirven como punto de referencia para un diagnóstico temprano de los costos de la reconstrucción en el posconflicto, son insuficientes para medir el impacto en términos de desarrollo y destrucción del tejido social.

El costo de la destrucción del capital social y la degradación institucional de la que son objeto las entidades sociopolíticas envueltas en un conflicto violento, son elementos cuya cuantificación es tan problemática como crucial para disminuir la probabilidad de entrar en un ciclo de violencia que pueda, eventualmente, reactivar una confrontación de escala igual o superior al conflicto inicial.

Para la construcción de una paz duradera, el análisis juicioso de los efectos del conflicto en los vínculos sociales y la inclusión de este punto en los esfuerzos de reconstrucción por parte de agentes gubernamentales y organismos internacionales, reviste una importancia capital.

En cuanto al marco de referencia histórico, un elemento clave es la finalización de la Guerra Fría, momento en que cambia la dinámica de los conflictos armados y por consiguiente, también la forma en que se ven afectadas las sociedades.

Durante la Guerra Fría, la intervención de fuerzas extranjeras en África, Asia y Centroamérica animadas por motivaciones geopolíticas impulsó unos niveles de sofisticación de la violencia sin precedentes, incluyendo el despliegue de fuerzas mercenarias y transferencia técnica y tecnológica que a posteriori, se convertirían en factores de desestabilización de largo plazo.

La terminación del conflicto ideológico Este-Oeste llegó acompañada de cambios fundamentales en la opinión pública internacional, apoyada en la capacidad de transmisión en tiempo real de nuevos medios de comunicación de alcance global que potenciaron la aversión del público especialmente en Occidente, al despliegue de tropas en territorio extranjero.

El fin de la intervención directa en África subsahariana, por ejemplo, dejó como resultado incontables caudillos militares desentendidos del derecho internacional; ejércitos sin una fuente oficial de ingresos o una autoridad que limitara su tendencia a convertirse en grupos mercenarios con alcance regional; un considerable arsenal y un vasto mercado de oferentes de municiones y equipo militar especialmente proveniente de la ex Unión Soviética.

En tales condiciones de ausencia de una autoridad central y de incentivos económicos para la actividad privada, sociedades devastadas económica y políticamente favorecieron la dinámica del saqueo y la expropiación, continuando o desatando nuevos conflictos civiles que consumieron más de 10 países de la región después de 1990. Toda esta situación es enmarcada por Kaldor en función de las nuevas guerras: 
Las nuevas guerras implican un desdibujamiento de las distinciones entre la guerra (normalmente definida como violencia por motivos políticos entre Estados o grupos políticos organizados), crimen organizado (la violencia por motivos particulares, en general el beneficio económico, ejercida por grupos organizados privados) y violaciones a gran escala de los derechos humanos (la violencia contra personas individualmente ejercida por Estados o grupos organizados políticamente (Kaldor, 2001, p.16)

Los cambios en estas nuevas guerras son identificados desde cuatro elementos: actores, objetivos, métodos y formas de financiación (Kaldor, 2013). Desde los actores, existe en primer lugar una erosión de la autonomía del Estado, específicamente en cuanto a la capacidad para mantener el monopolio de la violencia legítima.

El Estado está incapacitado para conservar el control del territorio, sumado a los efectos de gobiernos altamente corruptos y caracterizados por ser personalistas. Esta situación lleva a un proceso de privatización de la violencia que se explica por la aparición de múltiples unidades en combate que generan un tejido complejo de relaciones. Kaldor identifica fundamentalmente cinco tipos de actores armados: Fuerzas armadas regulares, Grupos paramilitares, Unidades de autodefensa, Mercenarios extranjeros y Tropas extranjeras regulares.

En cuanto a los objetivos, a diferencia de las antiguas guerras con objetivos geopolíticos e ideológicos, los objetivos de las nuevas guerras están en función del poder pero justificados en las identidades. "Reivindicaciones del poder basadas en una identidad concreta, sea nacional, del clan, religiosa y lingüística" (Kaldor, 2001, p.21). Kaldor critica cómo las posiciones tribalistas o nacionalistas son instrumentalizadas en función de la movilización política.

\section{TRANSFORMACIÓN DEL IMPACTO SOCIOECONÓMICO}

Como se ha mencionado, siguiendo al colapso del orden mundial de la Guerra Fría, los conflictos entre Estados se han hecho menos frecuentes, asistiendo a una escalada en los conflictos de carácter civil que hoy en día representan la mayoría de los conflictos alrededor del mundo (INFODEV, The World Bank, 2013).

En este nuevo contexto, el desinterés de las potencias clásicas se tradujo en una disminución acelerada en el tráfico de armas y recursos financieros hacia las zonas en conflicto, suponiendo un cambio en el comportamiento tanto de las sociedades como de los actores en conflicto (Mikailof, Kostner, \& Devictor, 2002).

Por ejemplo, en el caso del África Subsahariana, los conflictos armados pasaron de ser mecanismos perversos para tramitar reivindicaciones políticas y sociales, a ser un modus vivendi aceptable para varias generaciones. 
Como consecuencia, se afectó gravemente la capacidad de los países para atraer la inversión extranjera necesaria para la construcción de infraestructura de provisión de servicios básicos o el desarrollo de un mercado que genere empleo e incentivos económicos para actividades lícitas creando una trampa de pobreza, violencia y vulnerabilidad institucional.

Para la comunidad internacional los esfuerzos para el mantenimiento de la paz dejaron de tocar tangencialmente la problemática del desarrollo para convertirse en dimensiones complementarias, requiriendo esfuerzos similares en materia de planeación; asignación de recursos y espacio en la agenda internacional.

Los escenarios de los nuevos conflictos civiles en África, si bien no dejan lugar a generalizaciones dado el número de variables que hacen de cada uno un problema singular, comenzaron a exhibir algunos elementos recurrentes como el carácter cíclico de la violencia; la tendencia a convertirse en fenómenos regionales y la afectación severa de los vínculos sociales y las instituciones.

En el primer caso, los ciclos de violencia están asociados a la trampa de pobreza y debilidad institucional descrita anteriormente. En sociedades devastadas y sin un ambiente propicio para la inversión y la iniciativa privada, es común encontrar tres elementos de carácter económico: un PIB per cápita de tendencia negativa; mala distribución del ingreso y un alto nivel de dependencia de exportaciones de sector primario.

En tales circunstancias, los países en etapa temprana de posconflicto enfrentan una posibilidad del $44 \%$ de recaer en un escenario de violencia en los primeros cinco años de la recuperación (Collier, 2003).

El segundo elemento, relacionado con la dimensión transnacional que pueden tomar los conflictos y que genera ambientes de inestabilidad regional es indirectamente proporcional a la centralización de la autoridad y la legitimidad de las instituciones, siendo un punto crucial la capacidad de ejercer control en las fronteras.

En las zonas en conflicto, las fronteras son especialmente importantes en la medida en que son el escenario de varios fenómenos importantes para el desarrollo de las hostilidades.

En primer lugar, los grupos de desplazados que se dirigen hacia las fronteras para escapar de la violencia concentrada en las ciudades principales y otras posiciones estratégicas para las facciones en contienda, se convierten en un activo importante para grupos rebeldes.

Dadas las condiciones de desarraigo, los vínculos étnicos y religiosos son explotados como elemento de asociación a partir del cual la población desplazada puede unirse a la lucha armada directamente a través del reclutamiento o indirectamente, favoreciendo y conformando estructuras de apoyo en labores de logística y tráfico de elementos de guerra. 
Dado que muchas veces esos vínculos étnicos, religiosos y culturales trascienden las fronteras y que las zonas de conflicto son un escenario donde se generan incentivos económicos para actividades ilícitas, no es de extrañar que grupos en países vecinos a un conflicto armado resulten involucrados en el desarrollo del mismo.

Adicionalmente, si se tiene en cuenta que en el caso de los países africanos los conflictos armados en la década del 90 dejaron más de 300 millones de personas desplazadas o exiliadas (DFID, 2001), el efecto regional de los conflictos puede extenderse en el tiempo más allá del cese de las hostilidades en virtud de problemas sanitarios; el desarraigo que limita la iniciativa privada para emprender proyectos productivos; la afectación de los flujos de comercio y el consecuente aumento de la percepción de riesgo que ahuyenta la inversión.

El tercer elemento recurrente en los conflictos civiles posteriores a 1990, es la afectación severa de los vínculos sociales y la estructura institucional.

La percepción de ausencia de una autoridad central que garantice el ejercicio de los derechos y el cumplimiento de los contratos constituye una atmósfera de desconfianza e incapacidad de ejecución que interrumpe el funcionamiento del mercado y estanca la actividad económica.

Esta situación se ve reforzada por la erosión de aquellos constructos sociales, sistemas de confianza y credibilidad, asociaciones (formales e informales), y percepciones que construyen los sistemas de participación política y económica del día a día y que denominaremos en adelante Capital Social.

Su destrucción implica la desactivación de vínculos dentro y entre comunidades, generando desconfianza y desmontando incentivos para el emprendimiento de proyectos productivos y la realización de negocios debido a la incertidumbre sobre la propiedad, la ejecución de los contratos y el alcance de la administración de justicia.

\section{TRANSFORMACIÓN DE LOS CONFLICTOS ARMADOS}

Las ilustraciones 1 y 2 a continuación muestran el cambio en la tipología de los conflictos en el mundo y en África subsahariana respectivamente.

Se tuvieron en cuenta los eventos de conflicto en cada periodo de cinco años que registraron más de 1000 víctimas mortales en batalla.

Dichos eventos se encuentran caracterizados como Extra Sistémicos, cuándo un gobierno se enfrenta a un agente no-estatal por fuera de sus fronteras; Interestatales, cuando las facciones en contienda son dos o más estados; Civiles, cuando no hay participación de un estado diferente a aquel que enfrenta a un grupo rebelde dentro de sus fronteras; e Internacionalizado, cuando uno o más estados intervienen como apoyo de las facciones en conflicto (Gleditsch, 2002). 
La figura 1 muestra la tendencia ascendente de los conflictos civiles desde 1970, con una variación positiva entre la década de 1980 y 1990 del 5\%, mientras que los conflictos internacionalizados en el mismo periodo disminuyeron un $21 \%$, reflejando la caída en las intervenciones en el extranjero de las potencias con el final de la Guerra Fría.

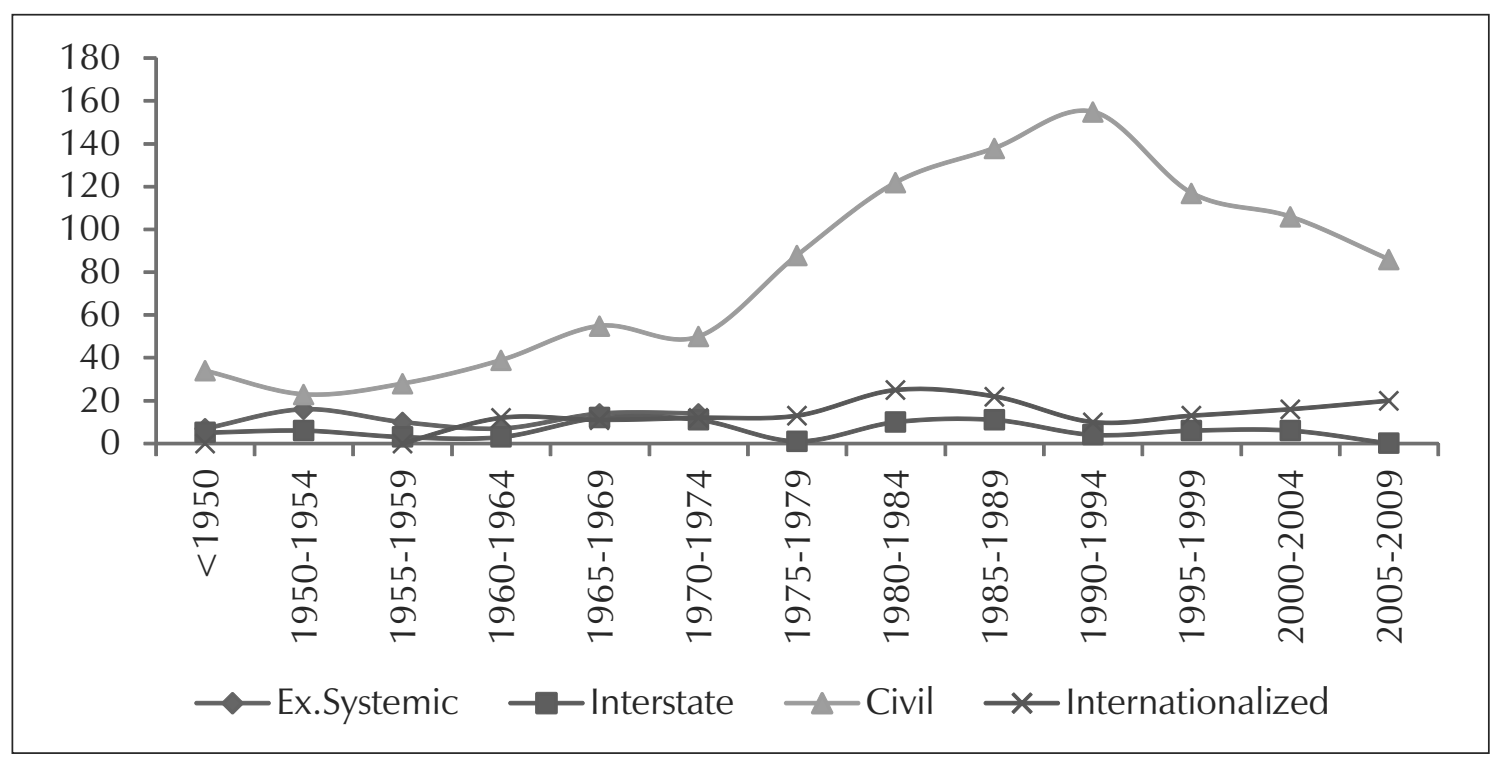

Figura 1. Tipos de conflicto en el mundo, 1950-2009

En el caso de África subsahariana, la figura 2 muestra la misma tendencia de manera exacerbada. Aunque se tratara de un fenómeno global, la ocurrencia de conflictos civiles en esta región del mundo creció un 21\% entre las décadas de 1980 y 1990 mientras que los conflictos internacionalizados disminuyeron en casi un $60 \%$. 


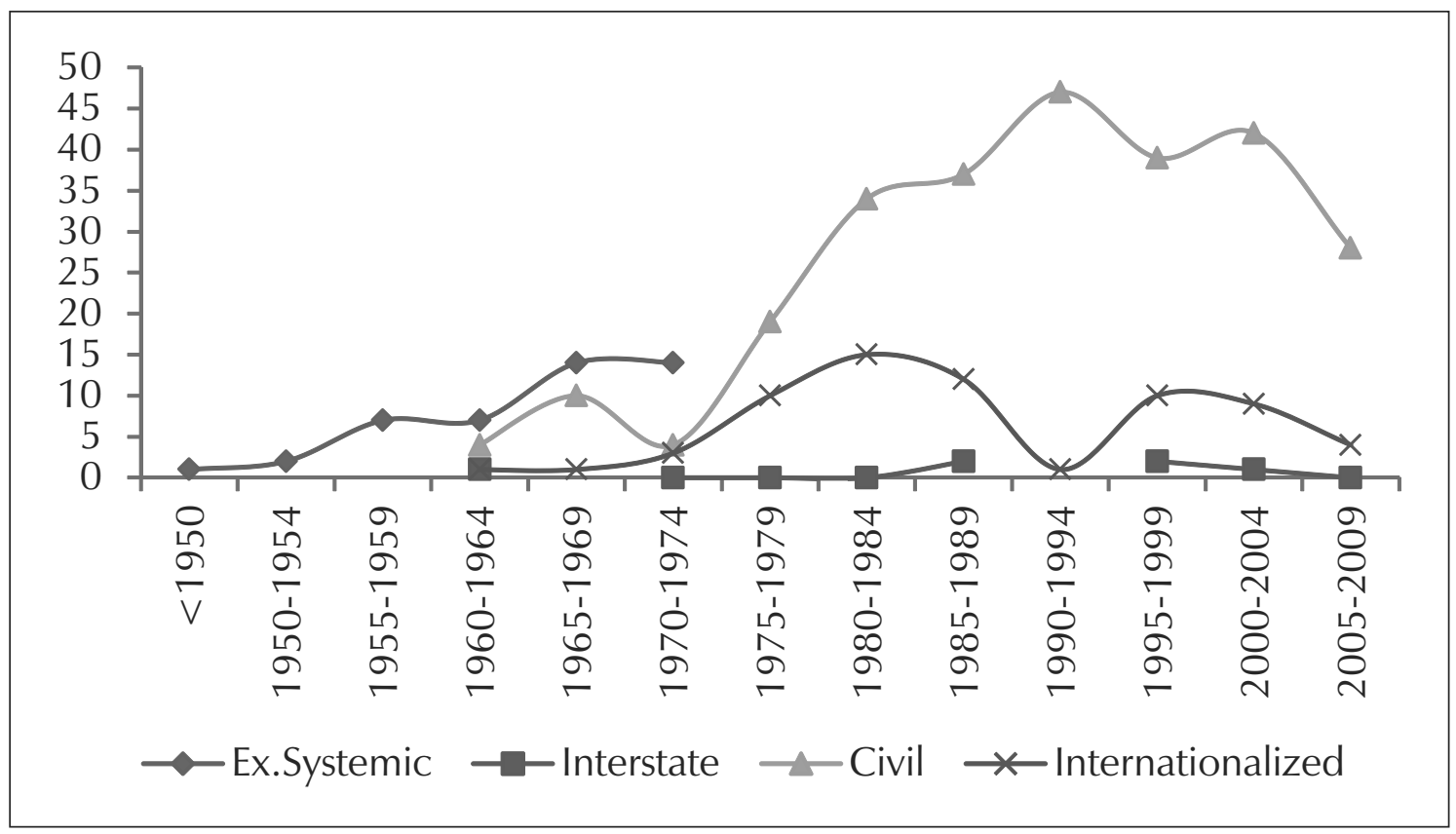

Figura 2. África Sub S., tipos de conflicto, 1950-2009

\section{DE LA GUERRA AL DESARROLLO: LA ETAPA DE TRANSICIÓN}

Uno de los grandes desafíos en el posconflicto es hacer del tránsito de la guerra al desarrollo un proceso irreversible. Lograrlo, además de importantes cantidades de recursos financieros, humanos y académicos, demanda el replanteamiento de los elementos institucionales y de política pública y económica que rigen las relaciones de la comunidad.

En los países donde los tres factores económicos negativos mencionados en el capítulo anterior convergen (PIB per cápita bajo; mala distribución del ingreso y dependencia de exportaciones de sector primario); enfrentar las causas raíz del conflicto puede resultar muy problemático de cara a las transiciones necesarias para el establecimiento de un sistema político y económico eficiente donde las tensiones sociales puedan tramitarse por vías no violentas.

Reconociendo los trascendentales procesos de recuperación de la seguridad y de reconciliación de las diferencias que el conflicto puede haber marcado en términos religiosos, étnicos o culturales, el presente análisis se enfoca en la transición económica por ser esta la de más largo plazo, siendo paralela a los procesos anteriores y proyectándose en el tiempo en el camino al desarrollo. 
Entre la economía de la guerra, caracterizada por fomentar redes clandestinas de búsqueda de rentas basadas en todo tipo de transacciones ilícitas y la economía del desarrollo, existe un terreno intermedio de reconstrucción y transición orientado a hacer la paz irreversible, mediante la búsqueda de herramientas que permitan establecer una economía funcional en donde además del acceso a servicios públicos básicos, las personas tengan acceso a fuentes lícitas sostenibles de ingreso y movilidad social (Castillo, 2012).

En esta fase, que coincide con las etapas más tempranas del posconflicto, el objetivo fundamental es establecer las bases de una recuperación que reduzcan la probabilidad de recaer en el conflicto armado.

Para lograrlo, los Estados en compañía de los agentes internacionales que participan del proceso de reconstrucción deben emprender una serie de tareas en varios frentes complementarios orientados a garantizar la seguridad de los ciudadanos, reconciliar facciones en conflicto e integrar ex combatientes y disminuir desequilibrios macroeconómicos.

Vale la pena aclarar aquí que la etapa posterior, denominada economía del desarrollo no implica que al final de la etapa de transición los Estados se encuentren preparados para comenzar un crecimiento sostenido en referencia al conjunto de variables con las que se identifica el desarrollo económico.

Simplemente, se espera que al final de la etapa de transición los Estados en recuperación librados del lastre de la guerra, cuenten con el aparato institucional y las herramientas de política económica y social para lidiar con los ciclos económicos y las tensiones sociales de manera adecuada.

En este sentido, una de las labores prioritarias es la estabilización de las relaciones políticas, económicas y sociales. Para ello es necesario el desarrollo de un marco institucional sólido, con cuerpos de seguridad, regulación y administración de justicia eficientes que permitan superar la acción de agentes desestabilizadores nacidos en el seno de la confrontación.

\section{ESTRUCTURAS CRIMINALES Y OTROS DESAFÍOS}

En los escenarios de conflicto armado, es posible encontrar estructuras organizacionales complejas, derivadas de la necesidad de los actores en conflicto de proveerse los mecanismos y los medios para la consecución de sus objetivos.

En tal sentido, emergen grupos interesados en el tráfico de material bélico, productos básicos, drogas, dinero y personas entre otros cuyo incentivo, por ser de carácter económico, persiste aun cuando la causa de conflicto desaparezca bien sea por la imposición de alguno de los actores o por el acuerdo pacífico entre las partes. 
Dichas estructuras evolucionan rápidamente y se adaptan al nuevo contexto, buscando nuevas fuentes de demanda y en el caso de las estructuras ilegales, potenciando la violencia urbana; el tráfico de drogas; la delincuencia común y el crimen organizado incluso en el nivel transnacional.

Los estudios realizados sobre procesos de postconflicto han documentado los riesgos más importantes que se presentan en estas situaciones y que amenazan la frágil paz en construcción. Paul Collier, Anke Hoeffler y Måns Söderbom argumentan que las sociedades en posconflicto tienen dos desafíos principales: la recuperación económica y la reducción del riesgo de recaer en violencia. El aumento de la violencia es un lugar común durante los procesos de paz y se determina en gran parte por la forma en las que se forman las nuevas instituciones y la forma en que fueron negociadas las condiciones de la paz (2006).

Además, establecen cómo esta violencia en el posconflicto trae tras de sí la imposibilidad de canalización y representación de los actores sociales y políticos, dando pie a la criminalidad dentro de las condiciones de polarización social. La diversificación de las formas de violencia hace poco posible el consenso social y establece la represión y la violencia misma como eje de las relaciones sociales (Collier, Hoeffler, \& Söderbom, 2006).

Esto tiene que ver con la débil gobernabilidad en muchas áreas proclives a la violencia, la cual ha deteriorado la seguridad del ambiente social debido a la carencia de autoridad estatal y al resultado de la transferencia de armas legales a facciones criminales y a una tradición de armar a las comunidades para enfrentar su propia inseguridad.

Robert Muggah refuerza esta posición demostrando que el periodo de postconflicto no es tan seguro como se cree: "La violencia armada, particularmente la cometida por ex soldados y las milicias informales, puede alcanzar proporciones epidémicas". Las armas usadas durante la guerra quedan a disposición del tráfico ilegal, lo que es determinante, para reforzar la criminalidad.

Muggah demuestra que el crecimiento del crimen organizado urbano después del conflicto es muy común. El crimen y la violencia social generalmente van en aumento después de los acuerdos de paz, incluso en términos más altos que los precedentes. Esta situación debe ser tenida en cuenta debido a que en muchos casos este efecto va más allá de las fronteras, se contagia a los vecinos incluso en Estados pacíficos. Bandas criminales y grupos milicianos crean coaliciones, incluso en el exterior, para el tráfico de drogas, contrabando y venta de armas (Muggah, 2005).

Berg (2005), trata de dar explicación a esta situación de la violencia en el postconflicto, argumentando que el desorden público y el aumento de la criminalidad y la violencia en el post conflicto se deben a cuatro factores: primero, actuaciones criminales del gobierno para mantenerse en el poder, utilizando ingresos provenientes del tráfico, el contrabando y otras actividades ilícitas para tal fin; segundo, el crecimiento de economías ilegales paralelas en donde surgen redes criminales, rutas y organizaciones transnacionales; tercero, las características del autoritarismo, en donde los gobiernos mantenían el orden por la fuerza y la intimidación a 
través de cuerpos de policía y servicios de seguridad; y finalmente, una sociedad dividida en donde surgen conflictos entre las facciones (Berg, y otros, 2005).

Al no tener un objetivo de carácter ideológico o político, estas estructuras criminales paralelas al conflicto son más flexibles que una fuerza armada, aprendiendo, innovando y adaptándose en periodos más cortos de tiempo. Cuentan además con un importante músculo económico que sustenta su supervivencia permeando las instituciones y las administraciones locales y regionales.

Con ello en mente, puede entenderse que la relación entre el Estado y un grupo armado ilegal es menos compleja que entre el mismo Estado y las estructuras criminales paralelas. Ésta última, puede darse en el contexto clásico de zonas de debilidad estatal o por la alianza con sectores corruptos de la función pública.

Para maximizar la eficiencia organizacional y evitar la acción del Estado, los criminales se adaptan al ambiente institucional existente, el cual está determinado principalmente por el Estado, el mercado y la estructura social (Xia, 2008). Dependiendo de la naturaleza del Estado, de la apertura del mercado y las características de la organización social, los grupos criminales identifican un nicho para sobrevivir y en consecuencia adoptan una forma de organización que les permita prosperar" (Garzón, 2012).

Además de los desafíos en materia de justicia; reparación de víctimas y reinserción a la vida civil de los excombatientes, las estructuras ilegales conexas que sobreviven al conflicto son otra dimensión de las preocupaciones del Estado y sus agentes en la etapa de transición. Identificarlas y desmantelarlas requiere esfuerzos que pueden demandar tantos recursos como el conflicto mismo y en muchas ocasiones, décadas de evolución en materia policial, institucional y de política pública.

Otro desafío importante en la etapa de transición de la economía de la guerra a la del desarrollo, es el de enfrentar algunos dilemas puestos por la necesidad de implementar herramientas institucionales y políticas de manejo de crisis.

Esto resulta problemático y puede generar resistencia de algunos actores debido a que este tipo de herramientas suponen esfuerzos financieros y priorizaciones que en principio podrían ir en contra de los tradicionales objetivos del desarrollo.

Efectivamente, las decisiones políticas en medio de la reconstrucción tienden a ser menos inclusivas, de corto plazo y altamente permeadas por miembros de la comunidad internacional amparados en los altos flujos de asistencia económica.

De nuevo, es importante resaltar que en esta fase de transición el objetivo principal es no recaer en el conflicto, antes que perseguir los objetivos tradicionales del desarrollo que, de cualquier forma, no pueden ser alcanzados si la paz no es sostenida (Castillo, 2012). 
De hecho, seguir la agenda para el desarrollo de un país en posconflicto es altamente riesgoso, pues deben considerarse las distorsiones producto de la ayuda financiera que pueden generar artificialmente tasas de crecimiento que no son sostenibles en el tiempo y no representan el mejoramiento de las condiciones de vida de las personas ni el mejoramiento de la capacidad operativa del Estado, otro factor crucial para la superación definitiva del conflicto.

\section{EL PAPEL DEL SECTOR PRIVADO}

La vinculación del sector privado al proceso de reconstrucción es un elemento cuya importancia puede parecer evidente pero que, sin embargo, con frecuencia queda por fuera de la mesa en las primeras etapas del posconflicto.

Esta situación se da por la conjunción de varios factores. Por ejemplo, las causas raíz del conflicto generalmente asociadas a elementos políticos, culturales, étnicos o religiosos se ven separadas del sector privado cuyo desarrollo es considerado propio de una etapa posterior.

Adicionalmente, los picos de ayuda financiera y la implementación de programas productivos con asistencia técnica proveniente de agentes internacionales en etapas tempranas del posconflicto, pueden crear distorsiones que impiden ver la necesidad de fomentar un clima de inversión atractivo para los particulares.

Estas situaciones desembocan en una dificultad posterior para incluir en las reformas propias de la reconstrucción, elementos tendientes al desarrollo del sector privado pues en el proceso pueden emerger intereses contrarios provenientes de grupos interesados en el control de la asistencia financiera o en la edificación de estructuras institucionales o de mercado que les sean favorables.

Sin embargo, la participación del sector privado en la elaboración de los marcos políticos en los que se desarrollarán los planes de reconstrucción es un elemento central en la transición de la guerra al desarrollo considerando, primero, la capacidad de dicho sector para suplir los vacíos técnicos y financieros propios de un Estado en recuperación (por ejemplo, en la provisión de servicios públicos o la generación de empleo) y segundo, el valor de las relaciones económicas que incentiva como mecanismo de construcción de capital social.

De hecho, las situaciones de conflicto tienden a causar un efecto de marginalización de la actividad empresarial debido a la ausencia de una autoridad o de una institución con la legitimidad suficiente para hacer practicable el marco regulatorio existente.

En tales circunstancias, el panorama empresarial en el posconflicto se caracteriza por la presencia de pequeñas empresas y negocios informales actuando en un escenario de alto riesgo y vulnerabilidad que lo hace altamente dependiente de redes sociales que llenan parte de los vacíos institucionales. (Langford, 2005) . 
Debido a la permanencia de necesidades básicas de provisión de bienes y servicios, que se hacen especialmente críticas durante un conflicto, los negocios y la inversión (aún informal y de pequeña escala) son algunos de los pocos puntos de contacto incluso entre las facciones en conflicto o entre grupos sociales afectos a bandos opuestos de la confrontación.

Bien sea por la búsqueda de rentas para el beneficio propio o actuando dentro de un tipo organizacional más complejo, las transacciones entre individuos pueden romper la dinámica de fragmentación propia del conflicto armado, creando redes de confianza que pueden ser aprovechadas durante la reconstrucción.

Ejemplos como el de la organización Dushirehamwe de mujeres Tutsis y Hutus en Burundi cuya orientación inicial era el diálogo para la reconciliación, y que terminó siendo un vehículo para actividades económicas conjuntas, es prueba del importante papel de los negocios en la reconstrucción del tejido social.

En este caso en particular, se hizo evidente la necesidad de superar obstáculos básicos para el desarrollo humano como la procura del sustento diario que se dio a partir de cooperativas agrícolas. Esto permitió a los grupos con una carga étnica asociada al conflicto encontrar objetivos comunes y crear puentes de cohesión social (Schwarts, Hahn, \& Bannon, 2004).

De otro lado, la reconstrucción de la infraestructura de transporte y telecomunicaciones contribuye a la recuperación de la confianza del público permitiendo a grupos desplazados restablecer vínculos familiares y con su lugar de origen. El retorno a los lugares de origen donde se tiene una menor percepción de vulnerabilidad al desarraigo y la expropiación, es fundamental para la creación de incentivos a la producción y el emprendimiento que difícilmente sucederán en situación de desplazamiento forzado (Mills \& Fan, 2006).

Otro punto importante a considerar dentro del rol del sector privado en la reconstrucción del capital social, es su impacto procedimental en las transacciones individuales. Durante un conflicto armado, especialmente en aquellos de carácter civil, uno de los sectores más afectados es la banca. Por su naturaleza, la infraestructura dedicada a las actividades financieras es blanco de diferentes grupos, siendo sujeto de depredación en la escalada del conflicto.

Adicionalmente, debido a que es un sector cuya actividad requiere una relación muy activa con un ente regulador central, en escenarios de debilidad institucional y pérdida de legitimidad, su función de intermediación financiera colapsa casi en paralelo con la autoridad del gobierno.

Un primer impacto masivo de esta situación es la interrupción de los medios formales de pago, con lo que se da un fenómeno de predilección por el efectivo que no solo contribuye a la marginalización de los negocios formales, sino que resulta muy conveniente para las economías clandestinas. 
Sin acceso a medios transaccionales ni a una infraestructura con la seguridad física para almacenar el efectivo, el volumen de los negocios decae contribuyendo a la dinámica del saqueo y la expropiación (Lister \& Pain, 2004).

\section{CONCLUSIÓN: UN MODELO DE TRANSICIÓN}

La consecución de un acuerdo de paz es apenas el comienzo de un proceso de recuperación social que abarca numerosos frentes, cada uno de los cuales conlleva una serie de desafíos en materia política, institucional y económica.

Entre ellos, pudimos identificar cuatro dimensiones clave en el proceso de transformar una economía de guerra, en una economía capaz de perseguir los objetivos del desarrollo. Estas dimensiones: Estabilización, Infraestructura, Compromiso público y Consolidación Institucional; tienen a su vez unas características específicas en relación con la urgencia, la intensidad, y la cronología de implementación de las tareas que las componen.

La urgencia, es medida en función de la criticidad de las tareas que componen cada dimensión, de cara al objetivo de reducir la probabilidad de reactivación del conflicto.

La intensidad, reúne todos los esfuerzos en materia de recursos financieros, humanos, técnicos, logísticos y políticos, requeridos para llevar a cabo dichas tareas de manera eficiente. El tiempo de implementación está asociado a la vinculación de las tareas a los objetivos de corto, mediano y largo plazo de los actores interesados en la reconstrucción.

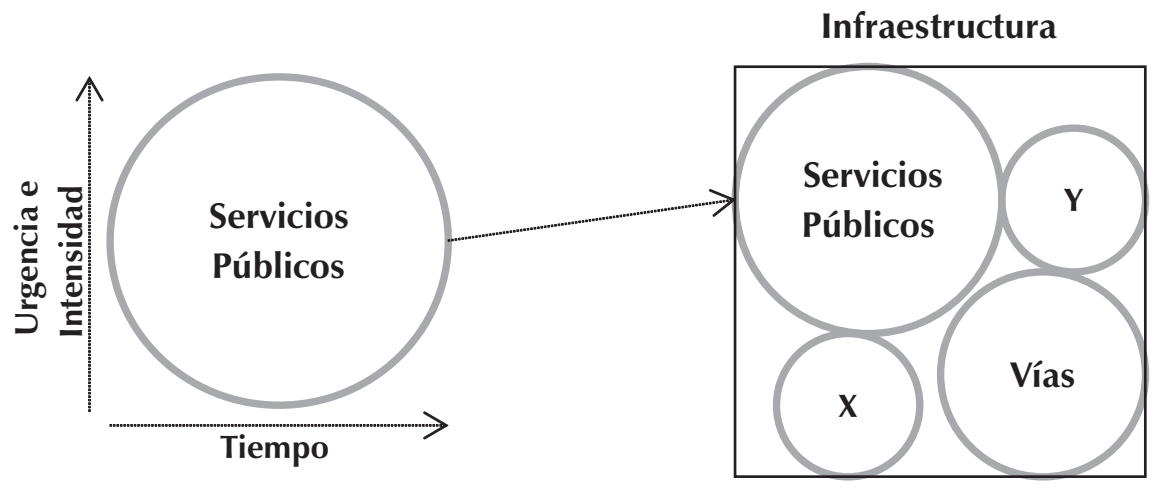

Figura 3. Dimensiones del modelo y sus variables.

La reconstrucción de Infraestructura de servicios públicos hace parte de la dimensión Infraestructura. Para cada dimensión se identifican las tareas primordiales y estas, a su vez, son valoradas en función de las características de Urgencia, Intensidad y Cronología definidas anteriormente. 
Luego, se trazó un modelo lineal sobre un plano dado por la evolución de la sociedad a partir del momento en que se da un acuerdo de paz, en las etapas de transición-reconstrucción y desarrollo (eje X); versus la evolución de las dimensiones en función de la acumulación de tareas.

Posteriormente, teniendo en cuenta el alcance temporal de los objetivos de los actores involucrados, se determinó la correspondencia de las tareas de corto, mediano y largo plazo con las fases de Reconstrucción-Transición y Desarrollo; completando así el escenario para trazar la pendiente que describe la evolución esperada de cada dimensión.

A continuación se ilustra el plano elaborado y se describen una a una las 4 dimensiones que serán trazadas en él:

Evolución de la dimensión

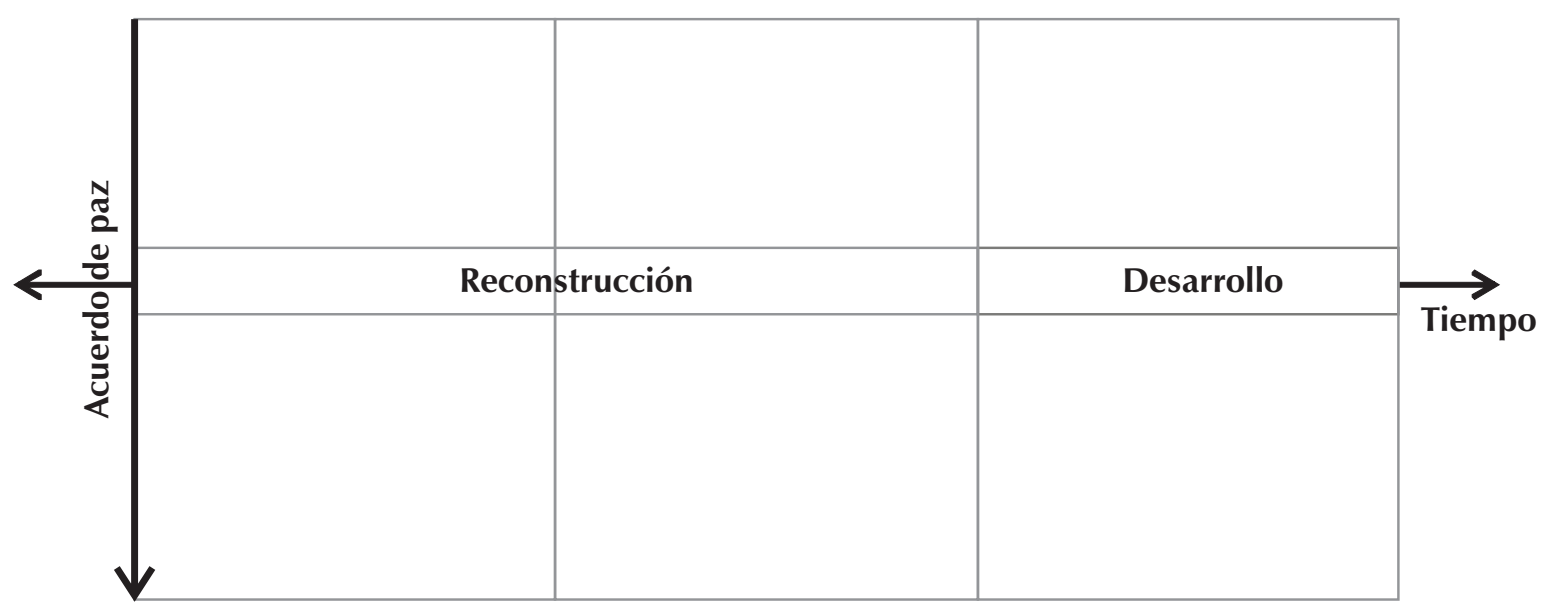

Figura 4. Plano de análisis

\section{DIMENSIÓN 1: ESTABILIZACIÓN}

En los conflictos civiles, una de las principales motivaciones está dada por la posesión de recursos estratégicos sobre la que compiten las facciones enfrentadas (Mikailof, Kostner, \& Devictor, 2002). Con el cese de las hostilidades, pese a que el acuerdo mismo puede incluir un marco regulatorio para el acceso a esos recursos, estos suelen ser motivo de disputa entre bandas criminales y otras estructuras persistentes especialmente en zonas de debilidad institucional o de difícil acceso para el control estatal.

Adicionalmente, la desmovilización de los combatientes sumada al desplazamiento forzado de las víctimas durante el conflicto, agudiza la problemática del desempleo y la baja generación de ingresos, dada por los escasos incentivos al emprendimiento y la iniciativa privada. 
Generación de empleo; mitigación de distorsiones macroeconómicas; creación de mecanismos eficientes para la desmovilización y posterior vinculación de ex combatientes al aparato productivo; así como el adecuado trámite de las disputas sobre recursos naturales y territorios, hacen parte de los desafíos logísticos y operacionales a los que se orientan las tareas de reconstrucción en la etapa de estabilización.

\section{DIMENSIÓN 2: INFRAESTRUCTURA}

Adicionalmente, la inversión privada juega un papel vital en la urgente reconstrucción de la infraestructura de provisión de servicios básicos. Esta no solo contribuye a la normalización de la actividad económica sino que reduce la posibilidad de tener escaladas de violencia por recursos escasos como el agua potable o la energía. Además, la rehabilitación de vías y medios de transporte y comunicación, resultan fundamentales para el retorno de la población desplazada a sus lugares de origen, donde tienen más incentivos para emprender proyectos productivos y evitar actividades informales e ilícitas para subsistir.

Vale la pena aclarar que la inversión en infraestructura conlleva la destinación de importantes recursos financieros, por lo que la percepción de riesgo limita la participación de capitales privados en los primeros 4 a 5 años de posconflicto. Con excepción del sector de Telecomunicaciones, primero en invertir en este tipo de escenarios, la participación privada en proyectos de infraestructura tiende a presentar una curva contraria al flujo de asistencia y ayuda internacional (Schwarts, Hahn, \& Bannon, 2004). De otro lado, la recuperación de medios transaccionales que limiten la preferencia por el efectivo y la flexibilización del marco regulatorio para hacer negocios, contribuyen a dinamizar el mercado que como se señaló, es uno de los puntos de contacto sociales más importantes durante y después del conflicto armado.

\section{DIMENSIÓN 3: COMPROMISO DEL PÚBLICO}

En cuanto al marco de las políticas públicas, es importante tener en cuenta que debido a los procesos de reparación y reconciliación donde deben atenderse grandes grupos de víctimas, las administraciones requieren tener la capacidad de implementar políticas poco incluyentes ante las que puede haber resistencia del público en el corto plazo. Estas políticas excluyentes deben flexibilizarse e irse desmontando en la medida en que la recuperación de la capacidad institucional permita el acceso equitativo a oportunidades políticas y económicas; lo que impactará positivamente el apoyo del público y la reconstrucción del capital social.

Estos últimos, son activos fundamentales que se mantienen apoyados en la confianza en las instituciones y la percepción de legitimidad de la autoridad. Las reformas que permiten recuperar la claridad en cuanto a la ejecución de los contratos y el alcance de la administración de justicia, son un incentivo a la iniciativa privada y fortalecen la capacidad de la comunidad para adoptar mecanismos alternativos de resolución de conflictos. 


\section{DIMENSIÓN 4: CONSOLIDACIÓN INSTITUCIONAL}

Una característica importante para identificar la finalización de la etapa de reconstruccióntransición, es la capacidad institucional para garantizar el acceso de la mayoría de la población a los servicios básicos. Ello implica unos niveles saludables de empleo; mecanismos de tributación eficientes; diversificación de las exportaciones; un ambiente propicio para la inversión privada y un marco regulatorio acompañado de una autoridad operativamente capaz de implementarlo en todo el territorio. Complementariamente, además del control exclusivo de la fuerza es importante que las labores de policía sean desempeñadas por cuerpos civiles no militarizados.

\section{MODELO PROPUESTO DE EVALUACIÓN DE LAS DIMENSIONES CLAVE EN EL POST CONFLICTO}

Con base en el análisis realizado, a continuación se expresa gráficamente la evolución obtenida para cada dimensión. Las características de demanda de recursos, criticidad y cronología de cada una de las tareas que las componen, resultan en la pendiente de cada dimensión:

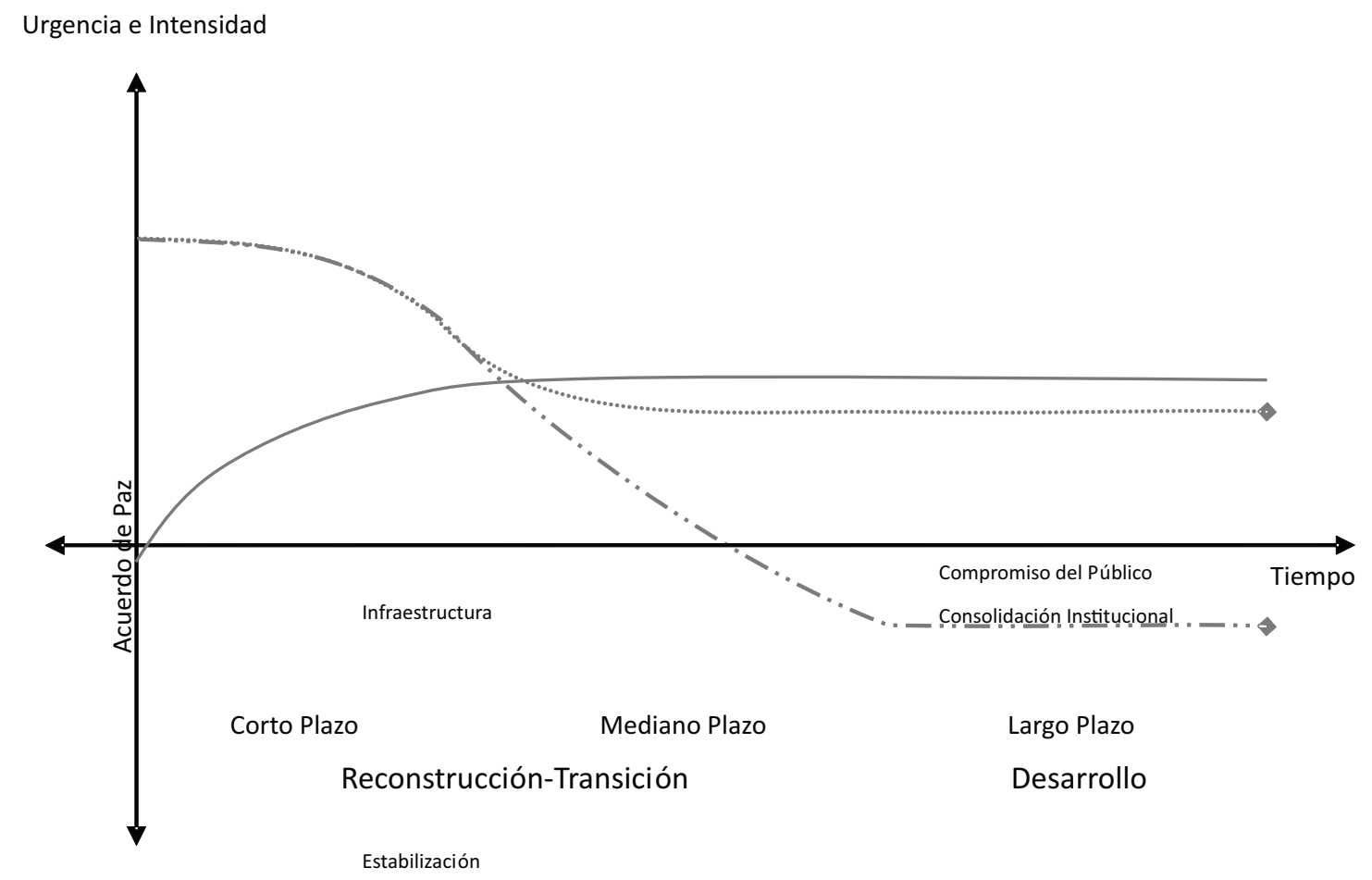

Figura 5. Evolución de dimensiones clave en el posconflicto.

En el modelo se ilustra con una pendiente negativa la dimensión de estabilización, (línea de guión y dos puntos) cuyas tareas tienen un enfoque de corto plazo y una demanda de recursos 
tendiente a cero a medida que se acerca al límite de la etapa de reconstrucción-transición. Esto se da debido a que se considera como requisito para entrar en la etapa de desarrollo un ambiente estable en materia económica y política.

Ligeramente por debajo se proyecta la curva de la dimensión de Infraestructura (línea punteada fina). Esta es paralela a la dimensión anterior en el corto plazo, pero luego su pendiente tiende a cero debido principalmente a que la provisión de servicios básicos es una necesidad transversal a las fases de transición y desarrollo.

Debido a que el punto cero del modelo coincide con el cese de las hostilidades, el compromiso del público (línea continua) tiene una pendiente positiva requiriendo importantes esfuerzos en el corto plazo para cumplir con las expectativas generadas por el acuerdo y lidiar con el descontento por el incumplimiento de compromisos. Además, el comportamiento de la dimensión es influido por el impacto de las tareas de estabilización con las que guarda una estrecha relación.

Posteriormente, su pendiente tien de a cero en el mediano y largo plazo debido a que la consolidación institucional requiere de un importante nivel de participación y confianza del público.

La Consolidación Institucional (línea de guiones) exhibe una pendiente positiva, siendo un objetivo de mediano y largo plazo que se hace crítico y prioritario en la medida en que las dimensiones precedentes se estabilizan. Dicha estabilización implica además la liberación de recursos para proyectos alineados con objetivos universales como aumento de la educación superior, cuidado del medio ambiente, desarrollo sostenible y políticas justas de comercio.

Una vez estabilizada la vida económica y política y establecidos los mecanismos de interacción social y participación adecuados; el proceso de consolidación institucional puede avanzar permitiendo al Estado abandonar la etapa de reconstrucción y encaminarse a la persecución de los objetivos del desarrollo en un escenario de normalidad.

\section{REFERENCIAS}

Acemoglu, D., \& Robinson, J. (2012). Why Nations Fail: The origins of power, prosperity and poverty. New York: Crown Publishing / Random House.

Berg, L.-A., Bhatti, Z., Bulkeley, J., Dunn, C., Lovejoy, J., Montgomery, M., Stene, A. (2005). The Missing Priority: Post-Conflict Security And The Rule Of Law. New Jersey: Report Prepared for the Office of Counterterrorism National Security Council The White House Washington, DC.

Castillo, G. d. (2012). Rebuilding war-torn states: Tomorrow's challenges for post-conflict reconstruction. Obtenido de Australian Civil Military Centre: http://acmc.gov.au/civil- 
military-working-paper-042012-rebuilding-war-torn-states-tomorrows-challenges-forpost-conflict-reconstruction/

Collier, P. (2003). Breaking the Conflict Trap: civil war and development policy. Washington, DC: World Bank Policy Research Report.

Collier, P. (2005). El desafío global de los conflictos locales. Bogota: Banco Mundial.

Collier, P. (2010). El club de la miseria. Qué falla en los paises más pobres del mundo. Bogota: Random House Mondadori.

Collier, P., Hoeffler, A., \& Söderbom, M. (2006). Post-Conflict Risks. Oxford: Centre for the Study of African Economies, University of Oxford

DFID (Marzo de 2001). The Causes of Conflict in Africa. Obtenido de Department for International Development: UK Government: http://webarchive.nationalarchives.gov. uk/+/http:/www.dfid.gov.uk/pubs/files/conflict-africa.pdf

Garzón, J. C. (Marzo de 2012). La rebelión de la redes criminales: El crimen Organizado en América Latina y las Fuerzas que lo modifican. Obtenido de Wilson Center: http://www. wilsoncenter.org/sites/default/files/Garzon.Rebellion.ESP_.pdf

Gleditsch. (2002). UCDP/PRIO Armed Conflict Dataset Codebook. Centre for the Study of Civil Wars, International Peace Research Institute, Oslo (PRIO)

INFODEV, The World Bank. (junio de 2013). The Role of Information and Communication Technologies in Post-Conflict Reconstruction. Obtenido de www.infodev.org: www. infodev.org/postconflict

Kaldor, M. (2001). Las nuevas guerras. Violencia organizada en la era global. Barcelona: Tusquets Editores.

(2013). In Defence of New Wars. Stability: International Journal of Security and Development, 2(1), 1-16.

Langford, B. (2005). The Private Sector and Growth in Fragile States: a Literature Review. Londres.: Department For International Development, United Kingdom.

Lister, S., \& Pain, A. (Junio de 2004). Trading in power: The politics of free markets in Afghanistan. Obtenido de Afghanistan Research and Evaluation Unit Briefing Paper: www.areu.org.af/ publications/areu\%20political\%20economy\%20Brief.pdf 
Mikailof, S., Kostner, M., \& Devictor, X. (Abril de 2002). World Bank. Obtenido de www. worldbank.org/afr/wps/wp30.pdf

Mills, R., \& Fan, Q. (Septiembre de 2006). The Investment Climate in Post Conflict Situations. Obtenido de World Bank: http://info.worldbank.org/etools/docs/library/235864/ postconflict_paper.pdf

Muggah, R. (2005). No Magic Bullet: A Critical Perspective on Disarmament, Demobilization and Reintegration (DDR) and Weapons Reduction in Post-conflict Contexts. The Round Table, 94(379), $239-252$.

Schwarts, J., Hahn, S., \& Bannon, I. (2004). The private's sector role in the provision of infrastructure in post conflict countries: Patterns and Policy Options. Obtenido de World Bank: http//rru.worldbank.org/paperslinks/open.aspx?id=6398

Xia, M. (2008). Organizational Formations of Organized Crime in China: Perspective from the State, Markets and Networks. Journal of Contemporary China, 17-54. 